Check for updates

Cite this: Mater. Chem. Front. 2020, 4, 1256

Received 15th January 2020

Accepted 17th February 2020

DOI: $10.1039 / \mathrm{d} 0 \mathrm{qm} 00031 \mathrm{k}$

rsc.li/frontiers-materials

\title{
Aggregation-induced emission of a 2D protein supramolecular nanofilm with emergent functions $\dagger$
}

\author{
Ruirui Liu, ${ }^{a}$ Jiangbo Jing, ${ }^{\mathrm{b}}$ Song Zhang, ${ }^{\mathrm{b}}$ Ke Wang, (D) ${ }^{\mathrm{c}}$ Bin Xu, (D) $*^{\mathrm{b}}$ \\ Wenjing Tian (D) ${ }^{b}$ and Peng Yang (D) *a
}

\begin{abstract}
Aggregation-induced emission (AIE) of a 2D protein supramolecular nanofilm exhibiting multiple functions is achieved for the first time at the air/water interface or on a solid surface at a timescale of several minutes. The mixture of lysozyme, tris(2-carboxyethyl)phosphine (TCEP) and 9,10-distyrylanthracene with two ammonium groups (DSAI) results in the rapid synthesis of a phase-transited lysozyme (PTL) AIE nanofilm, coating or ink from a neutral aqueous solution at room temperature. The multifunctionality of these waterborne biocompatible DSAI@PTL AIE materials shows some potential applications such as antibacterial and anti-counterfeiting for edible items or living creatures. This strategy combines the advantages of AIE with a 2D biopolymer suprastructure and provides an eco-friendly interfacial material with biological functions and applications. By introducing versatile AIE molecules with different functions and emission, the development of optically active biomimic materials with a wide range of applications could be opened up, such as multi-colour polymer coatings.
\end{abstract}

\section{Introduction}

Aggregation-induced emission (AIE) was discovered by Ben Zhong Tang in the study of 1-methyl-1,2,3,4,5-pentaphenylsilole, ${ }^{1}$ and, to date, a series of luminogens with aggregation-induced emission (AIEgens) have been applied in bio- or synthetic polymers by the polymerization of AIEgen-containing monomers or postpolymerization modification of polymers with AIEgens. ${ }^{2-4}$ This finding has further promoted advances in a variety of topics such as protein conformation change, protein fibrillation, enzyme activity, DNA synthesis, DNA sequencing, visualization of polymerization reactions, polymer self-assembly, response to multiple stimuli, and energy conversion. ${ }^{5}$ However, the introduction of AIEgens into 2D biopolymer film systems has not been reported, although recyclable biopolymer-derived 2D materials have offered multiple opportunities in various fields of surface modification, biomaterials, lithography, optics and electronics. ${ }^{6-10}$ The difficulties in synthesizing AIEgen@2D biopolymer materials probably lie in

\footnotetext{
${ }^{a}$ Key Laboratory of Applied Surface and Colloid Chemistry, Ministry of Education, School of Chemistry and Chemical Engineering, Shaanxi Normal University, Xi'an 710119, China. E-mail: yangpeng@snnu.edu.cn

${ }^{b}$ State Key Laboratory of Supramolecular Structure and Materials, Jilin University, Qianjin Street No. 2699, Changchun 130012, China.E-mail: xubin@jlu.edu.cn

'School of Pharmacy, Health Science Center, Xi'an Jiaotong University,

Xi'an 710061, China

$\dagger$ Electronic supplementary information (ESI) available: Additional figures and experimental details. See DOI: 10.1039/d0qm00031k
}

two aspects. Firstly, unlike self-assembled biopolymer structures in nature, the fabrication of a macroscopic 2D biopolymer material through economic supramolecular assembly remains a challenge, let alone the integration with AIEgens. ${ }^{2-5,11,12}$ Secondly, the lack of a method to chemically or physically encode AIEgens into a 2D array of biopolymer chains further constrains the development of AIEgen@2D biopolymer materials. ${ }^{1-3,13}$

Herein, we report sensitive binding of AIEgens to newlydeveloped amyloid-like suprastructures, which present the promising capability to synthesize highly emissive fluorescent $2 \mathrm{D}$ proteinaceous nanofilms, coatings and water-based inks. In contrast to classical ThT staining on amyloid structures, the present work suggests that 9,10-distyrylanthracene with two ammonium groups (DSAI) as a typical water-soluble AIEgen having acceptable antimicrobial capability and good biocompatibility ${ }^{14}$ could bind to amyloid-like aggregates of lysozyme to induce a stable AIE phenomenon. The resultant oligomer nanoparticles carrying DSAI AIEgens would further aggregate at the air/water interface to form a macroscopic fluorescent nanofilm, on the liquid/solid interface to form a reliable fluorescent coating on a substrate or in the bulk solution to form a fluorescent water-based ink (Fig. S1, $\mathrm{ESI} \dagger$ ). These materials take advantage of both AIEgens and amyloid-like structures typically including high photostability AIEgens to resist photobleaching and to give robust interfacial adhesion of amyloid-like structures on virtually arbitrary substrates. These features, in combination with the intrinsic biocompatibility of the proteinaceous nature, ensure the application 


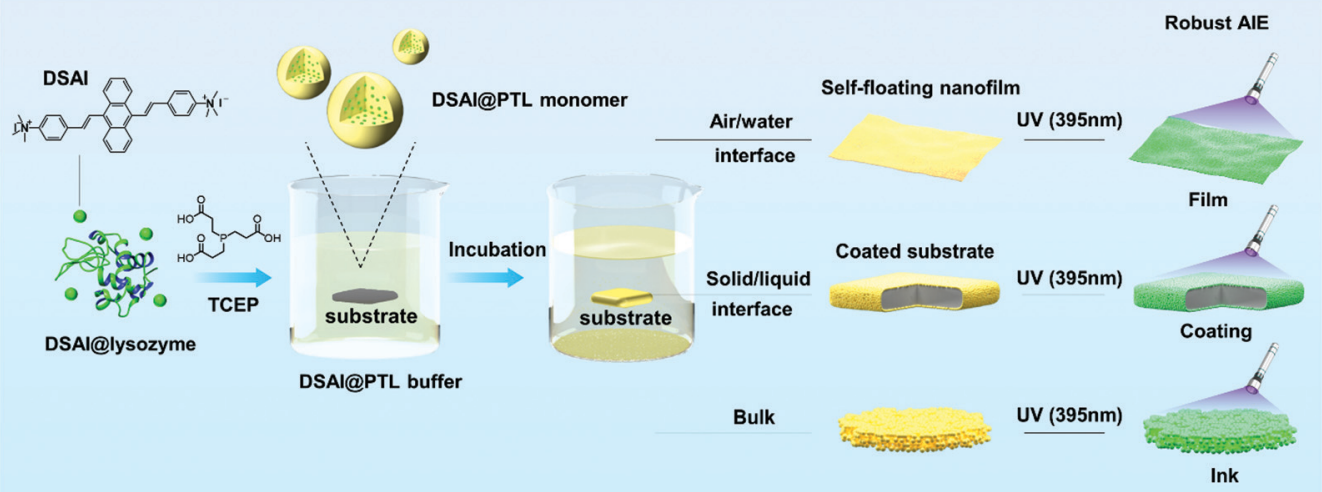

Scheme 1 Schematic illustration of the preparation of the DSAI@PTL coating (film and ink) on a substrate.

of this kind of hybrid material in the field of anti-counterfeiting on edible items and living creatures, which remains a huge challenge until now (Scheme 1).

The amyloid-like suprastructures, also called phase-transited lysozyme (PTL), including oligomeric nanoparticles and microparticles from protofibril aggregation, are prepared and characterized according to our previous study, ${ }^{15-17}$ in which lysozyme undergoes a fast phase transition upon the reduction of its disulfide bond by tris(2-carboxyethyl)phosphine (TCEP). Unlike classical amyloidosis to typically produce mature fibrils having long-range ordered $\beta$-sheet stacking, the PTL oligomeric nanoparticles having short-range ordered $\beta$-sheet stacking would aggregate at air/water or liquid/solid interfaces to form a nanofilm, and no mature fibrils are observed during the process. ${ }^{16}$ In the present work, the reaction for hybridizing DSAI with PTL is initiated by adding TCEP into a mixture of lysozyme and DSAI at a given condition ( $2 \mathrm{mg} \mathrm{ml}^{-1}$ lysozyme, $50 \mathrm{mM}$ TCEP, $\mathrm{pH}=7$, $0.1 \mathrm{mg} \mathrm{ml}^{-1}$ DSAI, volume ratio $10: 10: 2$, and incubation for 20-60 $\mathrm{min})$. The strategy to fabricate the fluorescent $2 \mathrm{D}$ hybrid proteinaceous nanofilm, coating and water-based inks for anticounterfeiting labels on substrate surfaces was then developed in a controllable manner (Fig. 1A-D). First, a self-floating hybrid nanofilm composed of a monolayer of oligomer nanoparticles was formed at the air/water interface of the reaction solution, and then the resultant film was further readily transferred onto the solid surface when the immersed material under the solution meniscus was lifted to pass through the air/water interface (Fig. 1A). Thus, the DSAI@PTL film is particularly useful for coating on a water-sensitive material. To accomplish this, the hybrid film was first formed at the air/water interface and then transferred onto an agarose hydrogel surface. Subsequently, the gel-carried film was stamped on a water-sensitive material surface for $\approx 10$ seconds to transfer the film from the gel surface onto the contacted material surface; the film was weakly attached to the gel surface, which is similar to the static-floating state on the water surface. By predefining the hydrogel shape, the film could also attach onto a material surface with a determined shape (Fig. 1A). Such patterning of the hybrid film allows it to have broad application prospects, for example, as a pattern design for the application of anti-counterfeiting described in an upcoming section. Alternatively, a similar 2D nanofilm composed of a monolayer of oligomer nanoparticles was directly formed at the liquid/solid interface when the material was immersed in the reaction mixture of lysozyme, TCEP and DSAI. The film coating was precisely immersion-guided, and the solution-dipped area was covered by the film (Fig. 1B). Further, with the use of the amyloid-like hybrid microparticles formed in the bulk solution as an ink, coating patterning could be also simply achieved by direct spraying or writing of the ink solution on a substrate (Fig. 1C and D). In this process, surface patterning was easily achieved by covering the substrate with a template during the spraying process from a watering pot or by directly writing on the substrate with a pen or brush.

\section{Results and discussion}

As revealed by the field emission scanning electron microscope (FE-SEM) cross-sectional images, the thickness of the nanofilm formed at the interfaces of air/water or liquid/solid could be adjusted to $20-50 \mathrm{~nm}$ by the concentration of lysozyme (Fig. 1E) and the $\mathrm{pH}$ of the TCEP solution (Fig. S2, ESI $\dagger$ ). ${ }^{16}$ Unlike the opaque or colorful properties of previously reported coating materials, ${ }^{18-20}$ the hybrid film formed at the interfaces showed a transmittance of $90-100 \%$ between 350 and $800 \mathrm{~nm}$ on the film-coated glass without an intrinsic color, thereby demonstrating potential as a unique class of stealthy coatings (Fig. $1 \mathrm{~F}$ and Fig. S3, ESI $\dagger$ ). The invisible coating was highly fluorescent under a $405 \mathrm{~nm}$ laser as the optimized excitation wavelength for DSAI $^{14}$ (Fig. 1G), while the control samples, including the blank PTL and blank DSAI solutions at the same concentration as that used in DSAI@PTL, did not emit obvious fluorescence (Fig. 1H, I and Fig. S4, ESI $\dagger$ ). This contrast indicated that the phase transition of lysozyme favored embedding and aggregation of DSAI in PTL to present AIE. The extent of the aggregation and resultant fluorescence emission could be further enhanced by increasing the concentration of DSAI, as normally observed in an AIE system (Fig. S5, ESI $\dagger$ ), ${ }^{14}$ or by increasing the pH of the TCEP buffer to intensify the extent of the phase transition (Fig. S6 and S7, ESI $\dagger$ ). ${ }^{16}$ The corresponding quantum yields 


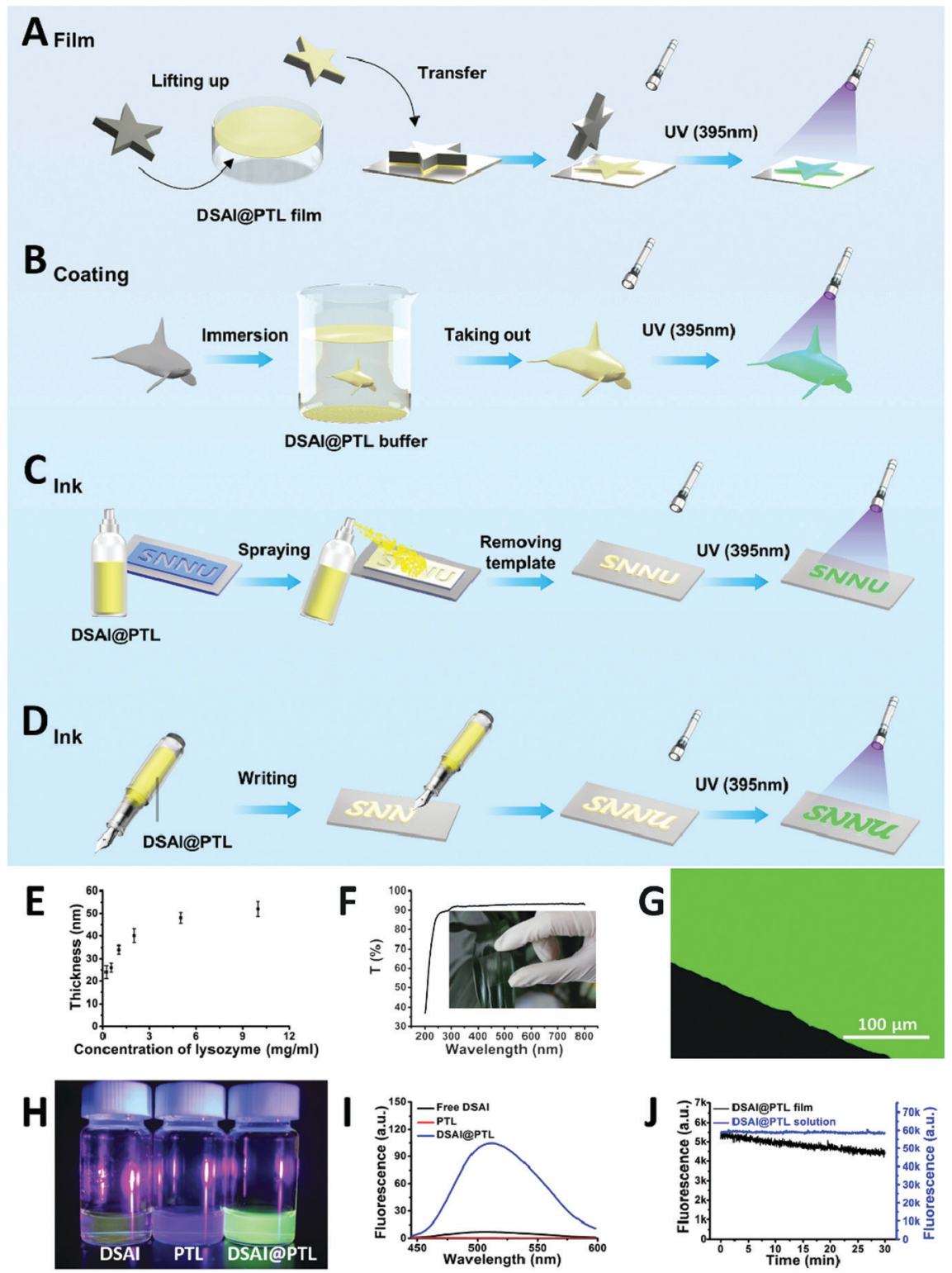

Fig. 1 The preparation of a self-floating 2D DSAI@PTL nanofilm and ink coating on a material surface. (A-D) Schematic strategies to form the DSAI@PTL nanofilm and ink coating on a material surface by (A) transfer from the liquid phase (contact-printing), (B) direct immersion, (C) spray coating and (D) direct writing. (E) The effect of the concentration of lysozyme on the thickness of the DSAI@PTL nanofilm. (F) UV/visible transmittance of the nanofilm coated on glass. (G) Confocal laser scanning images of the hybrid nanofilm under a $405 \mathrm{~nm}$ laser as the optimized excitation wavelength. (H) Fluorescence images and (I) corresponding spectra of free DSAI in water, free PTL in water and the DSAI@PTL hybrid in water under excitation with a $395 \mathrm{~nm}$ UV lamp (0.01 mg ml ${ }^{-1}$ DSAI was used in this case). (J) The time-resolved fluorescence spectra of the DSAI@PTL solution and hybrid film after exposure to a $340 \mathrm{~W}$ xenon lamp for $30 \mathrm{~min}$.

for the DSAI@PTL solution and hybrid film were 18\% and 11\%, respectively (Fig. S8, ESI $\dagger$ ). Due to the possible restriction of intramolecular rotations (RIR) of DSAI caused by the aggregated state of the molecules located in PTL, the DSAI@PTL solution and hybrid film resisted photobleaching when observed under a confocal microscope, and the corresponding fluorescence intensity after exposure to a $340 \mathrm{~W}$ xenon lamp for an extended time (30 min) changed slowly (Fig. 1J and Fig. S9, ESI $\dagger$ ). This anti-photobleaching capability is desirable for practical use.
Amyloid-based adhesion is a type of important bioadhesion in nature, which has been widely utilized by microbes and marine creatures to adhere themselves on a solid surface. ${ }^{21 a}$ Owing to the general and multiplex surface bonding affinity of a series of functional groups expressed on a PTL film surface to virtually arbitrary materials, ${ }^{21 b}$ the amyloid-like lysozyme assembly in the hybrid PTL film could produce robust interfacial bonding with various substrates, including inorganics, organics, metals, micro/nanoparticles and living organisms, like plants and animals. ${ }^{16,17}$ As a result, the direct formation of the DSAI@PTL 

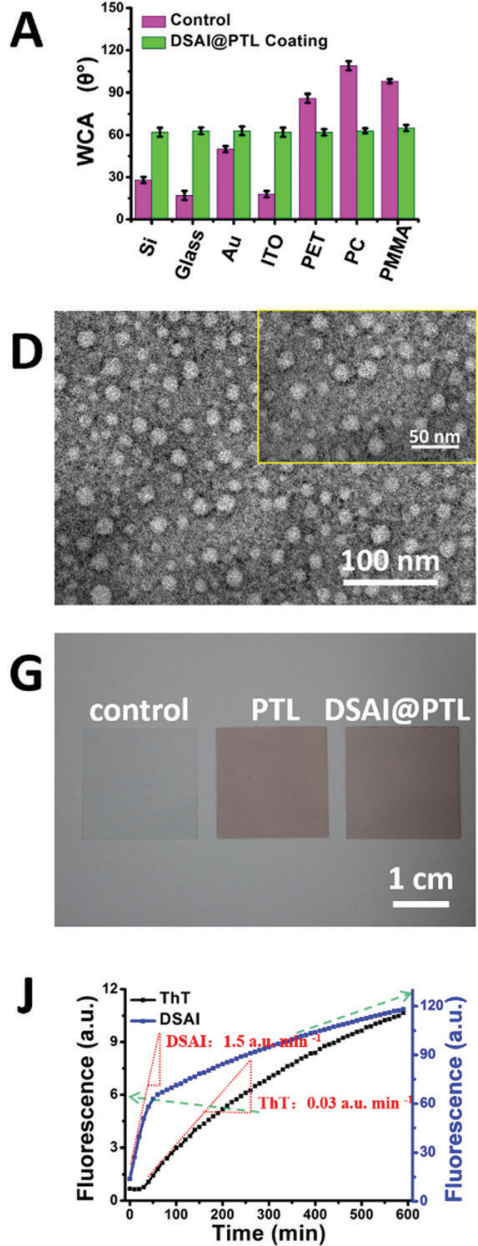
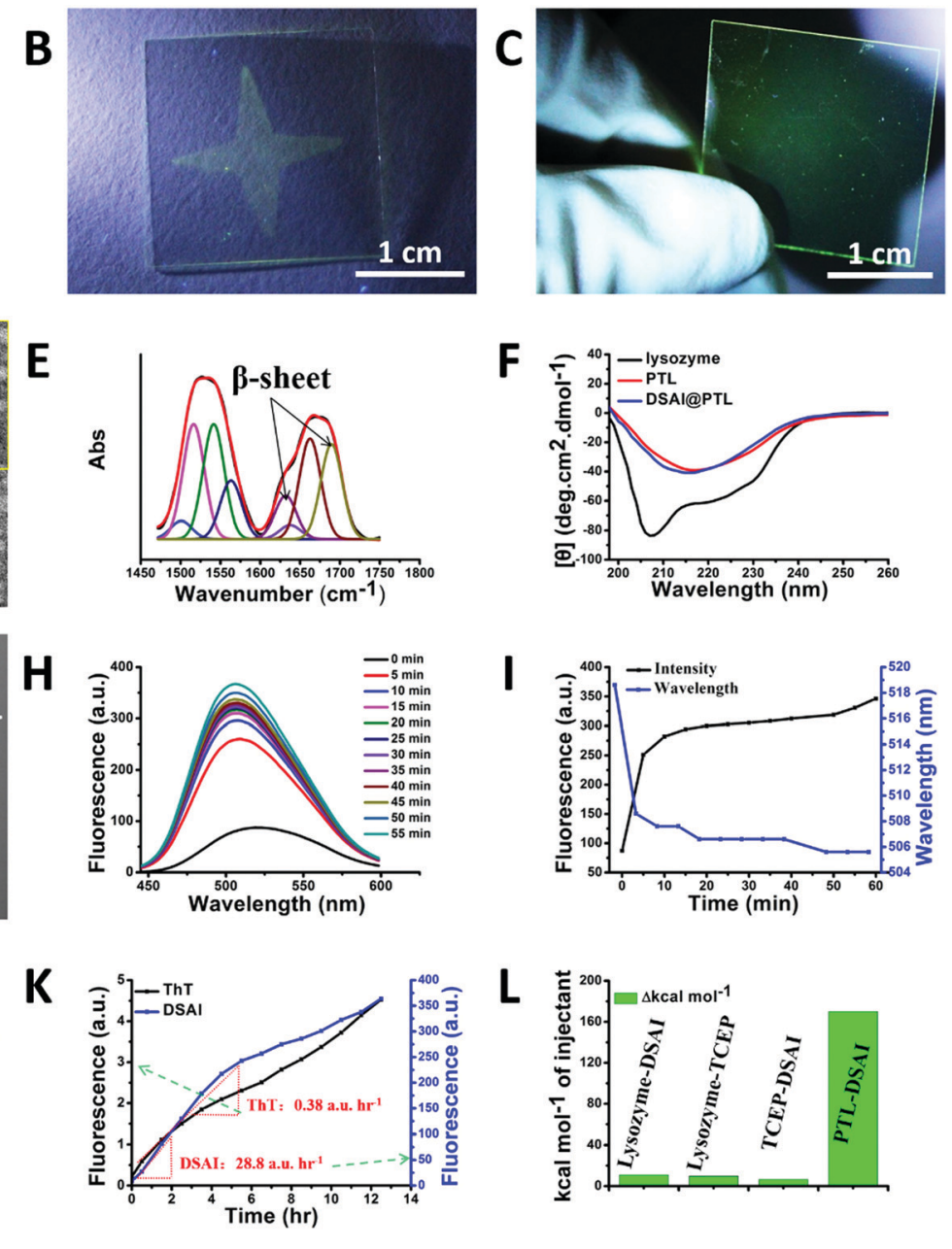

Fig. 2 (A) Bar graph representing the WCA of bare substrates and DSAI@PTL nanofilm-coated substrates. (B) Photograph displaying the fluorescence of star-shaped DSAI@PTL nanofilms under UV irradiation after $3 \mathrm{M}$ adhesive tape testing and ethanol treatment for $2 \mathrm{~h}$. (C) Photograph displaying the fluorescence of the DSAI@PTL nanofilm under UV excitation after ultrasonic treatment for $20 \mathrm{~min}$. (D) TEM image of the DSAI (aPTL nanofilm stained with $2 \%(\mathrm{w} / \mathrm{v}$ ) phosphotungstic acid for $5 \mathrm{~min}$. (E) The deconvolution of the amide I and II band of the hybrid nanofilm. (F) Far-UV CD spectra of the native lysozyme, PTL in solution (the pH of the TCEP buffer at 5.0, reaction time $2 \mathrm{~h}$ ), and DSAI@PTL in solution (the pH of the TCEP buffer at 5.0, reaction time $2 \mathrm{~h}$ ). (G) Photographs of a bare glass coverslip without any coating (control), glass coverslip with the PTL nanofilm coating (PTL) and glass coverslip with the DSAI@PTL nanofilm (DSAI@PTL) after Congo red staining. (H) The fluorescence spectra of DSAI@PTL evolving with the phase transition time. (I) The effect of the phase transition time on the fluorescence intensity and position of the maximum emission peak shown in ( $\mathrm{H}) .2 \mathrm{mg} \mathrm{ml^{-1 }}$ of lysozyme, $50 \mathrm{mM}$ of TCEP ( $\mathrm{pH} 7.5)$ and $0.01 \mathrm{mg} \mathrm{ml}^{-1}$ DSAl were used in these cases (volume ratio of lysozyme: TCEP:DSAI = $10: 10: 2$ ). (J) The fluorescence spectra of lysozyme $\left(2 \mathrm{mg} \mathrm{ml}^{-1}\right)$ evolving with the phase transition time $(50 \mathrm{mM}$ TCEP, pH 4.5) as revealed by staining the sample with ThT or DSAI. (K) Fluorescence spectra for the traditional amyloidosis of lysozyme $\left(2 \mathrm{mg} \mathrm{ml}^{-1}\right)$, as revealed by ThT staining $(100 \mu \mathrm{M})$ and DSAI (133 $\left.\mu \mathrm{M}\right)$. (L) ITC results for the interaction between DSAI and lysozyme, lysozyme and TCEP, DSAI and TCEP, and DSAI and PTL.

film on the substrates, including silicon wafers, metals, and polymers, was verified by simply incubating these surfaces in the lysozyme phase transition buffer with DSAI added, in which the versatile modified substrates covered by the DSAI@PTL film had a uniform water contact angle (WCA) of approximately $60^{\circ}$ (Fig. 2A and Fig. S10, ESI $\dagger$ ). The simultaneous exposure of polar and nonpolar groups on the PTL surface afforded multiplex interfacial bonding with the underlying substrate, ${ }^{21}$ which enabled the DSAI@PTL coating to resist mechanical peeling in the $3 \mathrm{M}$ adhesive tape peel test and ultrasonic treatment (Fig. 2B, C and Fig. S11, ESI $\dagger$ ). The robustness of the film on the substrate was also reflected by the thermostability of the coating under different temperatures, which showed an intact coating morphology and fluorescence intensity without obvious degradation after the film-coated glass slide was exposed to a temperature range from -20 to $200{ }^{\circ} \mathrm{C}$ (Fig. S12, ESI $\dagger$ ).

The long-term endurance of the film was further evaluated by an accelerated aging experiment, in which the film coating showed negligible changes in the surface morphology and fluorescence after being incubated in hot air at $80{ }^{\circ} \mathrm{C}$ and being exposed to ultraviolet irradiation or a microbial environment for 60 days (Fig. S13, ESI $\dagger$ ). The fluorescence intensity of the DSAI@PTL coating remained stable in a series of organic solvents, including ethanol, hexane, and DMF (Fig. 2C and Fig. S14, ESI $\dagger$ ), and then showed a 40\% (for the DSAI@PTL ink formed in the bulk solution) and $75-90 \%$ (for the DSAI@PTL 
nanofilm formed at the interface) decrease in an aqueous solution (Fig. S15, ESI $\dagger$ ). This phenomenon might be due to an environmentinduced migration of DSAI from the inside to the outside of the hybrid material, which was effectively overcome by crosslinking the DSAI@PTL coating in 2\% glutaraldehyde. This process then strengthened the coating and suppressed the diffusion of DSAI from inside the coating to the outside; as a result, stable fluorescence with a slight decrease was observed in polar and nonpolar solvents as well as extreme $\mathrm{pH}$ conditions, as characterized by the corresponding confocal microscopy images and fluorescence spectra (Fig. S16 and S17, ESI $\dagger$ ).

As revealed by transmission electron microscopy (TEM) and atomic force microscopy (AFM), the DSAI@PTL hybrid film comprised densely packed oligomer nanoparticles with an average diameter of approximately $30 \mathrm{~nm}$ (Fig. 2D and Fig. S18, ESI $\dagger$ ). ${ }^{16}$ The size of the oligomer nanoparticles was close to the nanofilm thickness, which also indicated a possible 2D monolayer distribution of oligomer nanoparticles inside the nanofilm. The amide I and II regions of the infrared (IR) spectra of the hybrid film indicated a contribution of antiparallel $\beta$-sheets in the film (Fig. 2E and Fig. S19, ESI $\dagger$ ). ${ }^{22}$ Consistently, the far-UV circular dichroism (CD) spectra of the reaction solution showed that the negative $\alpha$-helix peak of the native lysozyme at 208 and $222 \mathrm{~nm}$ was shifted to a single peak at $216 \mathrm{~nm}$ for the anti-parallel $\beta$-sheet structure after the phase transition (Fig. $2 \mathrm{~F}$ ), ${ }^{23}$ which was further supported by $\beta$-sheet-directed Congo red staining of the hybrid film (Fig. 2G). ${ }^{24}$ The hybridization process was then monitored by Thioflavin $\mathrm{T}$ (ThT) and 1-anilinonaphthalene-8-sulfonate (ANS) staining, which showed a successive increase in the fluorescence intensity of the reaction solution as the phase transition proceeded (Fig. S20, ESI $\dagger$ ). These results indicated that the transition process involved a continuous increase in the $\beta$-sheet structure, as identified by the $\beta$-sheet-specific dye ThT. ${ }^{29}$ The transition was assisted by the hydrophobic exposure and aggregation of the unfolded protein (after reduction of its intramolecular disulfide bond by TCEP), as reflected by hydrophobic residue-specific dye ANS. ${ }^{30}$ Interestingly, it was found that DSAI could also be utilized as a dye to monitor the phase transition process, indicating specific binding of DSAI to the $\beta$-sheet structure (Fig. S20, ESI $\dagger$ ). Unlike the slow fluorescence intensity increase at the beginning of the phase transition (Fig. S20, ESI $\dagger$ ) and the results of traditional amyloidosis revealed by ANS and ThT staining, ${ }^{23,25}$ the fluorescence intensity of the PTL system with DSAI (without addition of ANS and ThT) increased significantly from the beginning of the reaction, with the maximum emission peak shifting to a lower wavenumber (Fig. 2H-J and Fig. S20, ESI $\dagger$ ). This level of recognition was effective in a wide range of DSAI concentrations (e.g., from 0.01 to $1 \mathrm{mg} \mathrm{ml}{ }^{-1}$ ) (Fig. S21, ESI $\dagger$ ) and could be further utilized to monitor traditional amyloidosis by incubating lysozyme at a pH of 2 for $12 \mathrm{~h}$ (Fig. 2K). At roughly the same concentration, DSAI showed a much higher sensitivity than ThT from both the slope (50-75-fold increase) and the strength (10-100-fold increase) of the curve (Fig. 2J, K and Fig. S20, ESI $\dagger$ ). The above results reflected that DSAI-based AIE may provide a new method for monitoring conformational changes of proteins ${ }^{26-28}$ with a much higher sensitivity than the traditional ThT dye.
The fluorescence mechanism of DSAI@PTL was further studied by a series of experiments. First, because it is an amphiphilic molecule, the critical micelle concentration (CMC) of DSAI in pure water was measured in the $0.02-0.04 \mathrm{mg} \mathrm{ml}^{-1}$ range using its fluorescence spectra (Fig. S22, ESI $\dagger$ ). As a result, the DSAI in water at $0.01 \mathrm{mg} \mathrm{ml}^{-1}$ (or lower) typically utilized for hybridization with PTL would not substantially self-aggregate in the solution and thus not contribute to the strong fluorescence of DSAI@PTL (Fig. S4, ESI $\dagger$ ). Second, the native lysozyme was not capable of causing obvious fluorescence enhancement of DSAI, as reflected by the fluorescence intensity tests of the mixture solution of native lysozyme and DSAI (Fig. S23, ESI $\dagger$ ). Moreover, the strong fluorescence from the hybrid film was not mainly from the adsorption of the DSAI on the PTL product surface, since weak fluorescence due to the adsorption of DSAI on the as-prepared PTL surface would be easily diminished by simple desorption via a fast water rinse (Fig. S24 and S25, ESI $\dagger$ ). Based on the above results, it was hypothesized that the DSAI molecule was embedded within the short-range ordered $\beta$-sheet structure inside PTL, which then showed stable fluorescence after water or centrifugation rinsing as well as dialysis (Fig. S26, ESI $\dagger$ ). Such a model was then supported by isothermal titration calorimetry (ITC), in which the interaction between DSAI and PTL released substantially more heat (10-fold increase) than those of the interactions between DSAI and the native lysozyme, DSAI and TCEP or the native lysozyme and TCEP, indicating a specific interaction between DSAI and PTL (Fig. 2L and Fig. S27, ESI $\dagger$ ).

In addition to its fluorescent properties, the DSAI@PTL hybrid coating also showed good bactericidal function and biocompatibility, which would provide an important foundation for its use in the counterfeiting of biological species, foods and pharmaceuticals. The PTL and the DSAI moiety carrying two ammonium groups both contributed to the bactericidal function, which was more noticeable than that in the blank PTL film. ${ }^{21}$ High killing ratios of 99\% towards typical Gram-positive bacteria S. aureus (Staphylococcus aureus, ATCC 6538), Gram-negative bacteria E. coli (Escherichia coli, ATCC 8739), and fungus C. albicans (Candida albicans, ATCC 10231) were readily obtained through sufficient contact between the DSAI@PTL coating surface and the microbes (Fig. 3A and B).

Furthermore, in addition to the good intrinsic biocompatibility of the PTL film found previously by our group, ${ }^{31}$ a low cytotoxicity of DSAI towards mammalian cells at different concentrations was then shown using HL-7702 (normal liver cells) and HepG2 (liver cancer cells) as model cells, showing a weak influence on the cell viability (Fig. 3C). The survival curve of mice after direct feeding of DSAI at a maximum of $0.1 \mathrm{mg} \mathrm{ml}^{-1}$ (far higher than the dose used in the synthesis of the hybrid DSAI@PTL material) in a routine drug dose of $0.4 \mathrm{~mL}$ g@1 (mouse weight) exhibited a 100\% survival ratio until the end of the feeding period ( $>14$ days) (Fig. 3D). At the end of feeding, the histological evaluation of the group of mice fed with DSAI showed clear, neat and wellorganized cells in the heart, liver, spleen, lungs, kidneys, small intestine and colon as revealed by H\&E and Masson staining, which was consistent with the control group; bleeding, degeneration, hemorrhage, necrosis or inflammatory exudation 
A
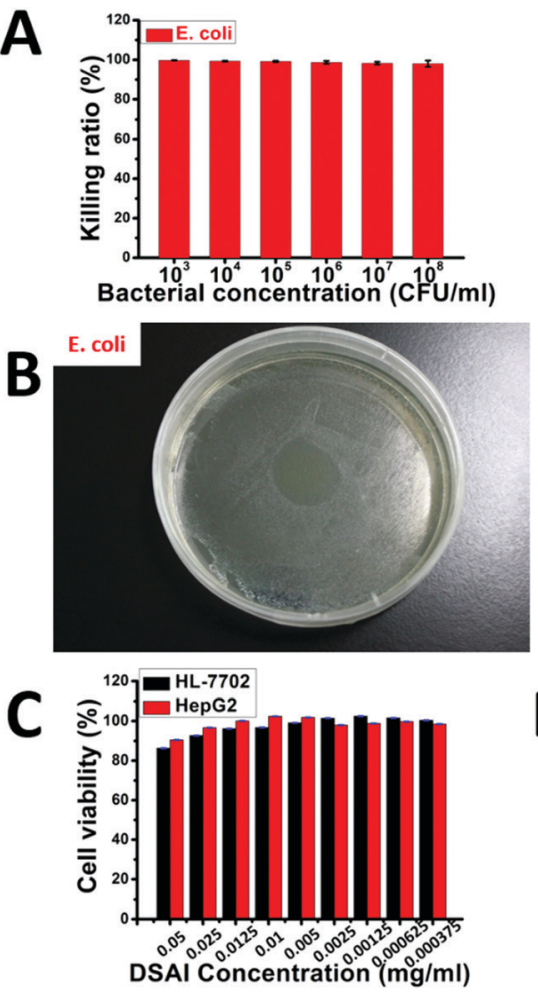
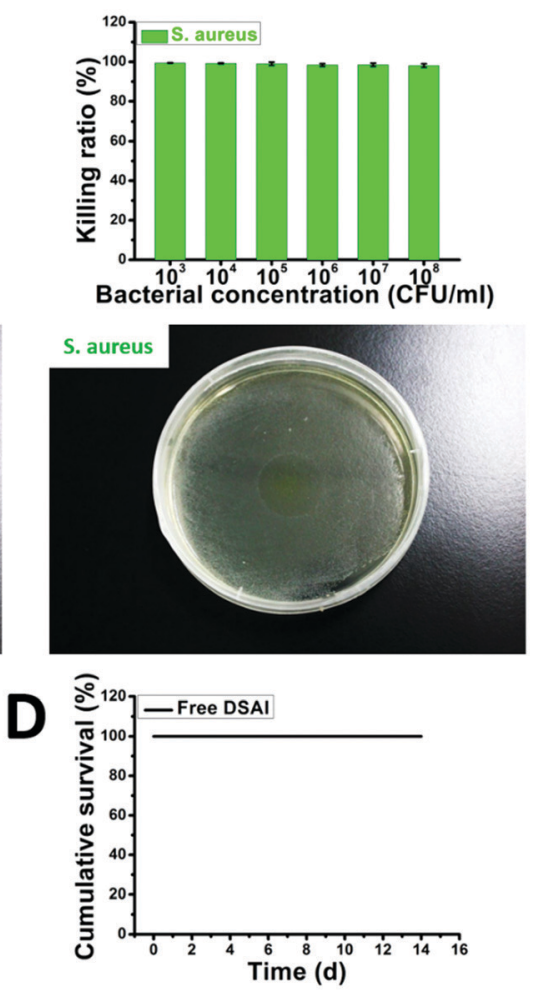
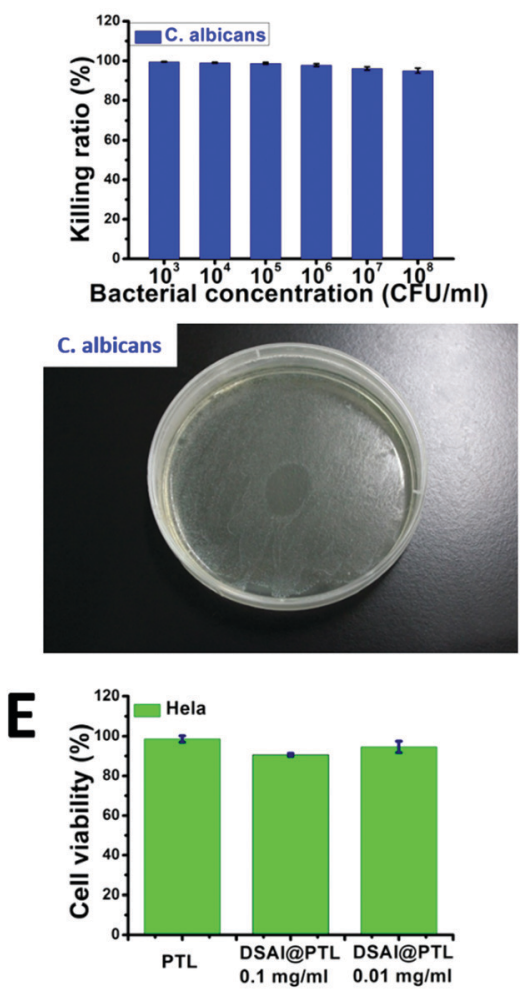

Fig. 3 Evaluation of the anti-microbial performance of the DSAI@PTL nanofilm toward E. coli, S. aureus, and C. albicans and the toxicity of the fluorescent ultrathin DSAICPTL nanofilm. (A) The killing ratio of the DSAI@PTL nanofilm toward different concentrations of E. coli, S. aureus, and C. albicans. The DSAI@PTL film was prepared by the strategy given in Fig. 1 B. $2 \mathrm{mg} \mathrm{ml}^{-1}$ of lysozyme, $50 \mathrm{mM}$ of TCEP (pH 7.5) and $1 \mathrm{mg} \mathrm{ml} \mathrm{m}^{-1}$ of DSAl were used in these cases. The volume ratio is $10: 10: 2$. (B) The corresponding plate spreading results of $E$. coli, S. aureus, and C. albicans treated with pure DSAI (0.01 mg ml-1). (C) The cytocompatibility of DSAI towards HL-7702 and HepG2 cells. (D) The survival curve of mice as a mammalian animal model by feeding $0.1 \mathrm{mg} \mathrm{ml}^{-1}$ DSAl to the mice. (E) The cytocompatibility of the DSAI@PTL coating towards HeLa cells. $2 \mathrm{mg} \mathrm{ml}^{-1}$ of lysozyme, $30 \mathrm{mM}$ of TCEP $(\mathrm{pH} 7.0)$, and $0.1 \mathrm{mg} \mathrm{ml}^{-1}$ or $0.01 \mathrm{mg} \mathrm{ml}^{-1}$ DSAl were used in these cases. The volume ratio was 1:1:0.2 and the incubation time was 20 min.

were also not observed (Fig. S28-S31, ESI $\dagger$ ). Based on the above results, a low cytotoxicity of the DSAI@PTL coating towards mammalian cells could be expected and was further confirmed using an MTT assay, which showed a weak influence on the viability and morphology of HeLa cells (Fig. 3E and Fig. S32, ESI $\dagger$ ). In this context, the hybrid material made of the pure proteinaceous substance and a low amount of DSAI, in combination with mild coating conditions (fast, room temperature, neutral aqueous solution), might show great potential in biological counterfeiting. These features are distinctive from those in conventional synthetic anti-counterfeiting systems, ${ }^{18-20}$ which is demonstrated in the following examples.

Counterfeiting is an increasing global challenge. In particular, counterfeiting in the fields of medicine and edible items can severely endanger human health. ${ }^{32-34}$ To combat counterfeiting, the packaging of an increasing number of goods is being protected by radio frequency tags, barcodes, watermarks, fluorescent inks, and chemical or biological tags (e.g., DNA) ${ }^{35-52,53}$ However, manufacturers often ship goods directly to wholesalers or distributors rather than consumers, which provides a means for counterfeit goods to enter the legitimate goods supply chain. Hence, labeling the goods themselves, such as tablets, instead of package labeling, could be a powerful strategy to protect consumers from fake goods. ${ }^{42,56}$ This idea was demonstrated using a DSAI@PTL coating. For instance, we simply wrote on glass with the DSAI@PTL ink, and there was no sign of the writing under ambient light, while the writing clearly appeared under ultraviolet light (Fig. S33, ESI $\dagger$ ). In another example, we easily transferred the patterned DSAI@ PTL hybrid film onto a tablet, which did not display any differences compared with the uncoated tablet under ambient light but there was obvious fluorescence under ultraviolet light (Fig. 4). It was further demonstrated that the DSAI@PTL nanofilm could readily adhere to various kinds of goods, such as capsules, foods, general merchandise, cherished animals and plants, precious artifacts, currency and colloids (e.g., living HeLa cells as shown in Fig. S32B (ESI $\dagger$ ) and $\mathrm{CaCO}_{3}$ microparticles as shown in Fig. S34 (ESI $\dagger$ ) due to the easy applicability of PTL onto micro/nano-particles ${ }^{17 b}$ ), which was invisible in ambient light but consistently displayed welldefined fluorescence signals under ultraviolet light (Fig. 4). A fluorescent anti-counterfeiting label could be easily erased by vitamin C (Fig. S35, ESI $\dagger$ ) as a result of specific insertion of vitamin $\mathrm{C}$ into the $\beta$-sheet inside the PTL leading to disassembly, which is likely a unique feature of DSAI@PTL. ${ }^{57}$ Overall, the above results proved that DSAI@PTL offers a convenient, effective, universal and reliable anti-counterfeiting strategy. 

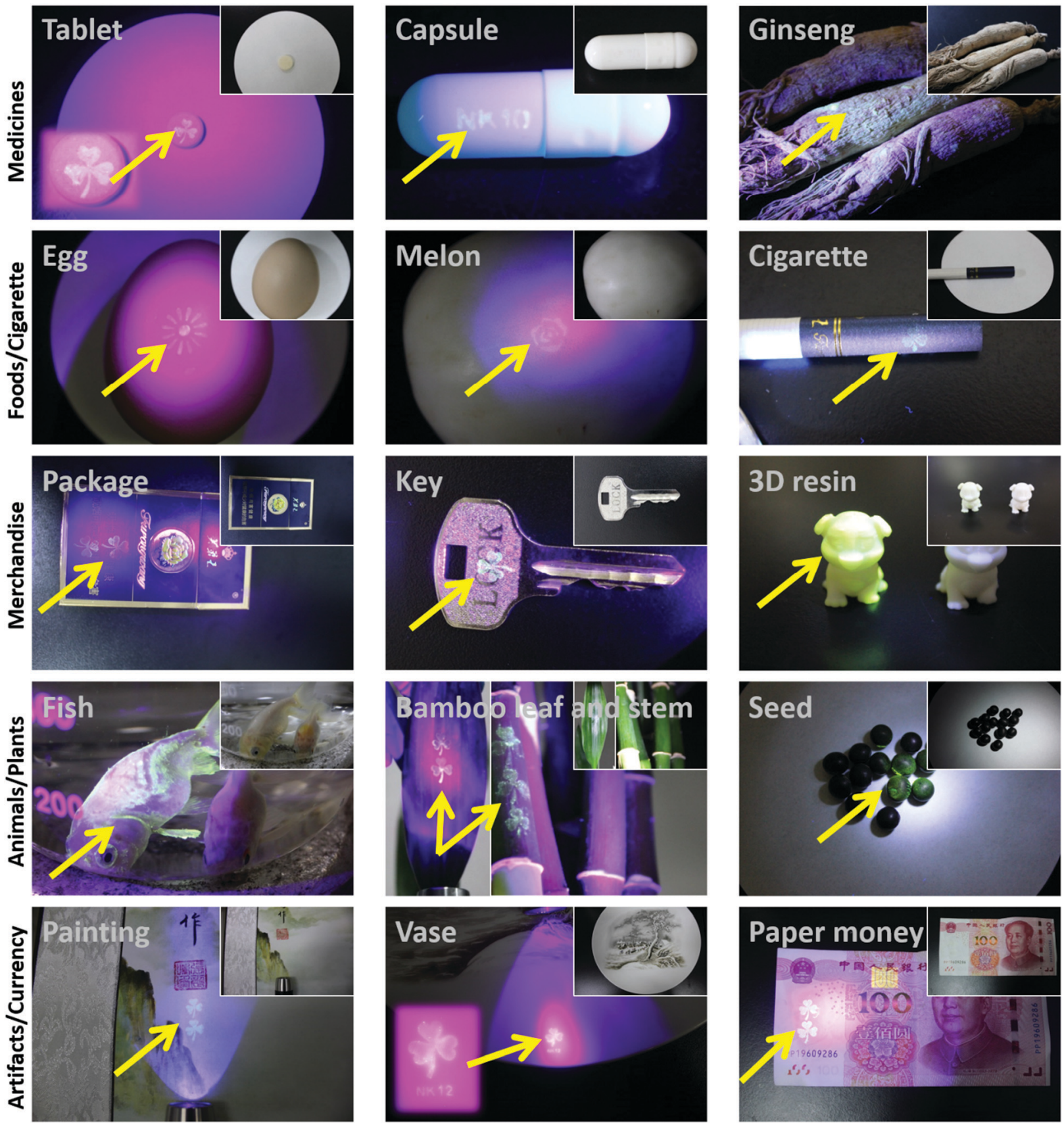

Fig. 4 The universality of the anti-counterfeiting strategy based on a fluorescent ultrathin DSAI@PTL nanofilm. Photographs under a UV lamp and ambient light (inset) for the DSAI (aPTL coating on medicines, foods, general merchandise, cherished animals and plants, precious artifacts and currency. For ginseng, 3D resin, fish and seed, a DSAI@PTL nanofilm without a pattern was formed on these surfaces; for other items, a nanofilm pattern was formed on the surfaces.

\section{Conclusions}

In conclusion, by combining the advantages of PTL (rapid and simple processing, straightforward operation and application at a large scale, low cost, high biocompatibility, being colorless and the ability to remain transparent under ambient light) with the advantages of an AIE molecule (sensitive to aggregation, good resistance to photobleaching, and strong fluorescence), we prepared a highly fluorescent amyloid-like ultrathin nanofilm and ink integrated with AIE luminophores with excellent stability by a simple one-step DSAI-doped amyloid-like assembly. The strong fluorescence of the DSAI@PTL film was attributed to the aggregation of DSAI embedded in the $\beta$-sheet-rich amyloid-like structure. The good interfacial adhesion of the DSAI@PTL nanofilm on the surfaces of various substrates, fluorescence stability, and robustness under a series of extreme conditions support its promising use in the anti-counterfeiting of various kinds of 
edible items and living creatures, such as microparticles (even living cells), general merchandise, medicines, fruits, foods, precious commodities, and living plants and animals.

\section{Experimental}

Materials

Lysozyme was purchased from Sigma-Aldrich. 4-(2-hydroxyethyl)-1-piperazineethanesulfonic acid (HEPES $\mathrm{pH}=7.2-7.4$, sterilized), MHB medium, YM medium polyimide (PI), phosphate buffered saline (PBS) and agarose powder were purchased from Solarbio. Tris(2-carboxyethyl)phosphine hydrochloride (TCEP) and Congo red were purchased from TCI. E. coli (ATCC 8739), S. aureus (ATCC 29213) and C. ablicans (ATCC 10231) were obtained from American Type Culture Collection (USA). Dulbecco's modified Eagle's medium (DMEM), penicillin-streptomycin solution, fetal bovine serum and trypsin were purchased from GE Healthcare Life Sciences. Ultrapure water was used in all experiments and was supplied by a Milli-Q Advantage A10 (Millipore, USA). Super flat glass (the grade for the electronics industry) and indium tin oxide (ITO)-coated glass plates were obtained from NanBo Co., Ltd. High-quality commercial poly(ethylene terephthalate) (PET) slabs, polycarbonate (PC) slabs and poly(methyl methacrylate) (PMMA) slabs with a protective film covering (for the nanofilm coating process, the protective film was carefully detached from the slab) were obtained from Taobao. Gold (45 nm Au with $5 \mathrm{~nm} \mathrm{Cr}$ as the adhesion promotion layer) coatings were prepared by magnetron sputtering deposition on cleaned silicon wafers. The PC slabs, PMMA slabs, PET slabs, gold wafers, glass and ITO-coated glass were subjected to ultrasonic cleaning with ethanol. The silicon wafers (Si) were purchased from Resemi Co., Ltd. The Si wafers and flat glass were cleaned with piranha solution (concentrated $\mathrm{H}_{2} \mathrm{SO}_{4}: 35 \% \mathrm{H}_{2} \mathrm{O}_{2}=7: 3 \mathrm{v} / \mathrm{v}$ ) at $80{ }^{\circ} \mathrm{C}$ for $8 \mathrm{~h}$ (Caution: piranha solution reacts violently with organic materials; it must be handled with extreme care, followed by copious rinsing with ultrapure water).

\section{The preparation of the DSAI@PTL buffer}

In order to obtain DSAI@PTL, the phase transition buffer of DSAI@lysozyme was freshly prepared by mixing the stock buffer of lysozyme $\left(2 \mathrm{mg} \mathrm{ml}^{-1}\right.$ in $10 \mathrm{mM}$ HEPES buffer at $\mathrm{pH}$ 7.2), DSAI (0.1 mg ml ${ }^{-1}$ dispersed in water) and TCEP buffer (50 $\mathrm{mM}$ TCEP in $10 \mathrm{mM}$ HEPES buffer at pH 5.0) at a volume ratio of $1: 0.2: 1$. Unless otherwise specified, the typical condition for the lysozyme buffer was $2 \mathrm{mg} \mathrm{ml}^{-1}$ lysozyme in $10 \mathrm{mM}$ of HEPES buffer, pH 7.2, and the typical condition for the TCEP buffer was $50 \mathrm{mM}$ TCEP in $10 \mathrm{mM}$ of HEPES buffer, $\mathrm{pH}=5.0$ (adjusting the $\mathrm{pH}$ with $5 \mathrm{M} \mathrm{NaOH}$ ). A lysozyme concentration higher than $2 \mathrm{mg} \mathrm{ml}^{-1}$ was not utilized since the concentration of $2 \mathrm{mg} \mathrm{ml}{ }^{-1}$ was enough to offer a dense and smooth nanofilm on a surface that had a $50 \mathrm{~nm}$ thickness and fully covered the underlying substrate. The assembly of lysozyme and DSAI was initiated sponteneously upon the mixing of them with TCEP added, and the DSAI@PTL product in the form of a 2D DSAI@PTL nanofilm was formed at the solution surface after 10-60 minutes.

\section{The attachment of the DSAI@PTL nanofilm via lift-up transferring}

The preparation strategy is given in Fig. 1A. In brief, the DSAI@PTL solution was dropped on a hydrophobic surface such as a Petri dish substrate to ensure a solution with a certain thickness. Then the solution on the substrate was incubated in a humid environment for a given time (generally for $60 \mathrm{~min}$ ) at room temperature. Then, a free-floating DSAI@PTL nanofilm that covered completely the whole solution surface area could be formed at the air/liquid interface. By carefully transferring the substrate holding the DSAI@PTL solution into a tank with ultrapure water inside, the DSAI@PTL nanofilm was then floated on the surface of the ultrapure water. The target substrate was lifted from the bottom of the tank to pass through the DSAI@PTL nanofilm. During this process, the DSAI@PTL nanofilm was inherently transferred onto the substrate surface. In the lift-up process, a pH higher than 5 in the TCEP buffer before mixing with DSAI@lysozyme was recommended since a $\mathrm{pH}$ lower than 5 easily produced a very thin nanofilm that was hardly observed by eye. The coated material was then dried at room temperature.

\section{The attachment of the DSAI@PTL nanofilm via in situ soaking}

The preparation strategy is given in Fig. 1B. Simple soaking is enough to transfer efficiently the DSAI@PTL nanofilm onto the immersed target substrate surface to form a solid-supported nanofilm. For this aim, the target material was fully contacted with the freshly prepared DSAI@PTL buffer, and after incubating for a given time (typically within 5-50 min) a conformal PTL nanofilm was stably attached onto the immersed solid surfaces. The coated material was then taken out and rinsed with clean water to wash away remaining native lysozyme and other salts. The coated material was then dried with nitrogen for the next purpose.

\section{The attachment of the DSAI@PTL nanofilm via contact-printing}

The preparation strategy is given in Fig. 1C. In this method, the DSAI@PTL nanofilm was firstly floated on the ultrapure water surface, and then transferred onto an agarose gel (prepared by dissolving $1 \%$ wt agarose in boiling water and cooling the solution to room temperature at ambient conditions) surface with a defined shape by the lift-up transferring technique (2.3). For transferring the DSAI@PTL nanofilm onto a target substrate, the DSAI@PTL nanofilm-coated gel stamp was evenly touched with the underlying substrate, and after a gentle pressure on the stamp for 5 seconds, the gel stamp was carefully peeled off from the substrate. In this way, the DSAI@PTL nanofilm orignally carried by the gel stamp would be transferred onto the underlying target substrate.

\section{The attachment of the DSAI@PTL nanofilm via direct writing or spraying}

The preparation strategy is given in Fig. 1D. In this method, the DSAI@PTL buffer could be written with using a writing brush or sprayed on the substrate through a watering pot and after that the DSAI@PTL nanofilm could be patterned easily through 
a template. For this aim, the freshly prepared DSAI@PTL solution was infused into a pen or a spray bottle, and then one can use the pen to write any security label on a substrate surface with a designed pattern. By placing the template on the substrate to be modified, one can use a spray bottle to spray any type of security label on the substrate. In this process, due to the rapid phase transition process, a patterned DSAI@PTL nanofilm could be formed on the substrate after spraying. If the substrate is water-resistant, the reaction residue can be washed away by ultra-pure water. In this method, a pH maintained at 5 in the TCEP buffer before mixing with DSAI@lysozyme $\left(2 \mathrm{mg} \mathrm{ml}^{-1}\right.$ lysozyme, concentration of DSAI dependent on requirements as show in Fig. S4, ESI $\dagger$ ) was recommended since under this condition the system will maintain a stable and non-separated state for several days.

\section{Cell viability assay}

Firstly, DSAI solutions with different concentrations were prepared: $0.1 \mathrm{mg} \mathrm{ml}{ }^{-1}, 0.05 \mathrm{mg} \mathrm{ml}^{-1}, 0.025 \mathrm{mg} \mathrm{ml}^{-1}, 0.0125 \mathrm{mg} \mathrm{ml}^{-1}$, $0.01 \mathrm{mg} \mathrm{ml}^{-1}, 0.005 \mathrm{mg} \mathrm{ml}^{-1}, 0.0025 \mathrm{mg} \mathrm{ml}^{-1}, 0.00125 \mathrm{mg} \mathrm{ml}^{-1}$, $0.000625 \mathrm{mg} \mathrm{ml}^{-1}$, and $0.000375 \mathrm{mg} \mathrm{ml}^{-1}$. HL7702 and HepG2 cells in the logarithmic growth stage were taken, washed with PBS, digested with trypsin, and centrifuged for $5 \mathrm{~min}$ at $1000 \mathrm{rpm}$, and the supernatant was removed. After adding the appropriate medium and blending it into a single-cell suspension, the cell liquid was transferred into a $15 \mathrm{ml}$ centrifuge tube and centrifuged for $5 \mathrm{~min}$ at $1000 \mathrm{rpm}$. After centrifugal cleaning, the medium was added to make it a single-cell suspension. The cells contained in the single cell suspension were counted with a blood cell count plate, and the cell density was regulated to $5 \times 10^{4} \mathrm{ml}^{-1}$. After the cells were adhered to the wall, we set the blank group, control group and experimental group. After culture for $24 \mathrm{~h}$, the supernatant was discarded, and $200 \mu \mathrm{L}$ with a final concentration of $0.5 \mathrm{mg} \mathrm{ml}^{-1}$ MTT was added to each well. After incubation for $4 \mathrm{~h}$ for the culture plate at $37{ }^{\circ} \mathrm{C}$ and $5 \% \mathrm{CO}_{2}$, the supernatant was abandoned and inject $150 \mu \mathrm{l}$ DMSO in each group, followed by the oscillation for 10 min with avoiding light. Finally, the incubated solution was studied using a spectrophotometric microplate reader (BioRad 680, USA) at a wavelength of $492 \mathrm{~nm}$. The MTT assay was also used for HeLa cells, PTL coated HeLa cells and DSAI@PTL coated HeLa cells.

\section{The toxicity evaluation for DSAI by recording the survival curve of Kunming mice}

The experiment was divided into four groups. There were 10 mice in each group, including 5 males and 5 females. Before feeding chemicals, the mice were forbidden to eat for 4-5 h. After that 0.01 and $0.001 \mathrm{mg} \mathrm{ml}^{-1}$ DSAI solution were respectively intragastrically administered according to $0.4 \mathrm{ml} / 20 \mathrm{~g}$ (body weight of the mice) every day. Every physiological behavior of the mice was observed within $1 \mathrm{~h}$ after injection of the DSAI solution. Finally, the mice were switched to a healthy diet at $2 \mathrm{~h}$ after the dosing. We recorded the number of deaths in the mice every day. After 14 days of research, the heart, liver, spleen, lungs, kidneys, small intestine and colon were subjected to histological evaluation.

\section{Characterization}

The Attenuated Total Reflectance Fourier Transform Infrared (ATR-FTIR) spectrum was recorded on a Vetex 70v spectrometer (Bruker Inc., Germany). The UV/vis spectrum was collected using a U-3900/3900H (Hitachi). The fluorescence spectrum was collected using an FLS-920 fluorescence spectrophotometer (Edinburgh Instruments). Scanning electron microscopy (SEM) was performed using an FEI Quanta 200. Field Emission SEM (FE-SEM) observations were conducted on an SU8020 (Hitachi). Atomic Force Microscopy (AFM) was performed using a CSPM 5500 (MultiMode, NanoScope IV from Benyuan Inc, China) and silicon cantilevers with resonance frequencies of $f 0=300 \mathrm{kHz}$ and spring constants of $k=40 \mathrm{~N} \mathrm{~m}^{-1}$ were used. The nanofilm coating on the silicon wafer was scanned by using the tapping mode, and the mean diameter and root mean square (RMS) roughness were analyzed using software CSPM Imager 4.7. For Transmission Electron Microscopy (TEM) measurements, the DSAI@PTL nanofilm was deposited on a copper grid (with a carbon support film) by the strategy given in Fig. 1B and further negatively stained with $30 \mu \mathrm{l}$ of $2 \%$ (w/v) phosphotungstic acid aqueous solution $(\mathrm{pH}$ 7.0) for about $5 \mathrm{~min}$. Then, we let it naturally dry for TEM measurements. TEM was performed on a JEM-2100 (JEOL, Japan) at $200 \mathrm{kV}$. Particle size distribution measurements of the colloids were performed by Dynamic Light Scattering (DLS) on a Malvern Zeta sizer Nano-ZS90. Laser confocal microscopy was performed on an OLYMPUS FV1200. Optical microscopic observations were carried out on a Nikon Ti-U (Tokyo, Japan). The water contact angle (WCA) was measured on an OCA 20 (Dataphysics, Germany). The far-UV CD spectrum was collected by using a Chirascan spectrophotometer (Applied Photophysics Ltd, England).

\section{Conflicts of interest}

The authors declare that they have no conflict of interest.

\section{Acknowledgements}

P. Y. and B. X. are thankful for the funding from the National Natural Science Foundation of China (no. 21875132, 51673112, 21835001), the 111 Project (no. B14041), the Shaanxi Science Fund for Distinguished Young Scholars (2187JC-018), the Fundamental Research Funds For the Central Universities (GK201801003, 2017CBY004) and the Open Project of the State Key Laboratory of Supramolecular Structure and Materials (No. sklssm2019032).

\section{Notes and references}

1 J. D. Luo, Z. L. Xie, J. W. Y. Lam, L. Cheng, H. Y. Chen, C. F. Qiu, H. S. Kwok, X. W. Zhan, Y. Q. Liu, D. B. Zhu and B. Z. Tang, Aggregation-induced Emission of 1-methyl-1,2,3,4,5pentaphenylsilole, Chem. Commun., 2001, 1740-1741.

2 R. Hu, Y. Kang and B. Z. Tang, Recent advances in AIE polymers, Polym. J., 2016, 48, 359-370. 
3 (a) Y. Hong, J. W. Y. Lam and B. Z. Tang, Aggregationinduced Emission: Phenomenon, Mechanism and Applications, Chem. Commun., 2009, 4332-4353; (b) Y. Hong, J. W. Y. Lamab and B. Z. Tang, Aggregation-induced Emission, Chem. Soc. Rev., 2011, 40, 5361-5388; (c) J. Mei, N. L. C. Leung, R. T. K. Kwok, J. W. Y. Lam and B. Z. Tang, Aggregation-Induced Emission: Together We Shine, United We Soar, Chem. Rev., 2015, 115, 11718-11940.

4 J. Mei, Y. Hong, J. W. Y. Lam, A. Qin, Y. Tang and B. Z. Tang, Aggregation-Induced Emission: The Whole Is More Brilliant than the Parts, Adv. Mater., 2014, 26, 5429-5479.

5 Y. Li, S. Liu, T. Han, H. Zhang, C. Chuah, R. T. Kwok, J. W. Y. Lam and B. Z. Tang, Sparks fly when AIE meets with polymers, Mater. Chem. Front., 2019, 3, 2207-2220.

6 H. Ejima, J. J. Richardson, K. Liang, J. P. Best, M. P. van Koeverden, G. K. Such, J. Cui and F. Caruso, One-step assembly of coordination complexes for versatile film and particle engineering, Science, 2013, 341, 154-157.

7 J. D. Brodin, J. R. Carr, P. A. Sontz and F. A. Tezcan, Exceptionally stable, redox-active supramolecular protein assemblies with emergent properties, Proc. Natl. Acad. Sci. U. S. A., 2014, 111, 2897-2902.

8 J. F. Matthaei, F. DiMaio, J. J. Richards, L. D. Pozzo, D. Baker and F. Baneyx, Designing two-dimensional protein arrays through fusion of multimers and interface mutations, Nano Lett., 2015, 15, 5235-5239.

9 F. Sakai, G. Yang, M. S. Weiss, Y. Liu, G. Chen and M. Jiang, Protein crystalline frameworks with controllable interpenetration directed by dual supramolecular interactions, Nat. Commun., 2015, 4, 4634.

10 H. S. Jang, J. H. Lee, Y. S. Park, Y. O. Kim, J. Park, T. Y. Yang, K. Jin, J. Lee, S. Park, J. M. You, K. W. Jeong, A. Shin, I. S. Oh, M. K. Kwon, Y. I. Kim, H. H. Cho, H. N. Han, Y. Kim, Y. H. Chang, S. R. Paik, K. T. Nam and Y. S. Lee, Tyrosinemediated two-dimensional peptide assembly and its role as a bio-inspired catalytic scaffold, Nat. Commun., 2014, 5, 3665.

11 B. Saif, W. Zhang, X. Zhang, Q. Gu and P. Yang, SnTriggered Two-Dimensional Fast Protein Assembly with Emergent Functions, ACS Nano, 2019, 13, 7736-7749.

12 T. P. J. Knowles, T. W. Oppenheim, A. K. Buell, D. Y. Chirgadze and M. E. Welland, Nanostructured films from hierarchical self-assembly of amyloidogenic proteins, Nat. Nanotechnol., 2010, 5, 204.

13 D. Chen, J. Zhan, M. Zhang, J. Zhang, J. Tao, D. Tang, A. Shen, H. Qiu and S. Yin, A fluorescent supramolecular polymer with aggregation induced emission (AIE) properties formed by crown ether-based host-guest interactions, Polym. Chem., 2015, 6, 25-29.

14 (a) X. Li, K. Ma, S. Zhu, S. Yao, Z. Liu, B. Xu, B. Yang and W. Tian, Fluorescent Aptasensor Based on Aggregationinduced Emission Probe and Graphene Oxide, Anal. Chem., 2013, 86, 298-303; (b) L. Ma, B. Xu, L. Liu and W. Tian, A Label-free Fluorescent Aptasensor for Turn-on Monitoring Ochratoxin A Based on AIE-active Probe and Graphene Oxide, Chem. Res. Chin. Univ., 2018, 34, 363-368; (c) K. Ma, H. Wang, X. Li, B. Xu and W. Tian, Turn-on Sensing for $\mathrm{Ag}^{+}$
Based on AIE-active Fluorescent Probe and Cytosine-rich DNA, Anal. Bioanal. Chem., 2015, 407, 2625-2630.

15 P. Yang, Direct Biomolecule Binding on Nonfouling Surfaces via Newly Discovered Supramolecular Self-Assembly of Lysozyme under Physiological Conditions, Macromol. Biosci., 2012, 12, 1053-1059.

16 (a) D. Wang, Y. Ha, J. Gu, Q. Li, L. Zhang and P. Yang, 2D Protein Supramolecular Nanofilm with Exceptionally Large Area and Emergent Functions, Adv. Mater., 2016, 28, 7414-7423; (b) C. Li, L. Xu, Y. Y. Zuo and P. Yang, Tuning Protein Assembly Pathways Through Superfast Amyloid-like Aggregation, Biomater. Sci., 2018, 6, 836-841; (c) F. Tao, Q. Han, K. Liu and P. Yang, Tuning Crystallization Pathways through Mesoscale Assembly of Biomacromolecular Nanocrystals, Angew. Chem., Int. Ed., 2017, 56, 13440-13444.

17 (a) A. Gao, Q. Wu, D. Wang, Y. Ha, Z. Chen and P. Yang, A Superhydrophobic Surface Templated by Protein SelfAssembly and Emerging Application Toward Protein Crystallization, Adv. Mater., 2016, 28, 579-587; (b) R. Liu, J. Zhao, Q. Han, X. Hu, D. Wang, X. Zhang and P. Yang, OneStep Assembly of a Biomimetic Biopolymer Coating for Particle Surface Engineering, Adv. Mater., 2018, 30, 1802851.

18 (a) D. Yan, J. Lu, M. Wei, S. Qin, L. Chen, S. Zhang, D. G. Evans and X. Duan, Heterogeneous Transparent Ultrathin Films with Tunable-Color Luminescence Based on the Assembly of Photoactive Organic Molecules and Layered Double Hydroxides, Adv. Funct. Mater., 2011, 21, 2497-2505; (b) R. Liang, S. Xu, D. Yan, W. Shi, R. Tian, H. Yan, M. Wei, D. G. Evans and X. Duan, CdTe Quantum Dots/Layered Double Hydroxide Ultrathin Films with Multicolor Light Emission via Layer-by-Layer Assembly, Adv. Funct. Mater., 2012, 22, 4940-4948; (c) Y. Qin, J. Shi, X. Gong, Z. Tian, P. Zhang and J. Lu, A Luminescent Inorganic/Organic Composite Ultrathin Film Based on a 2D Cascade FRET Process and Its Potential VOC Selective Sensing Properties, Adv. Funct. Mater., 2016, 26, 6752-6759; (d) R. Tian, S. Zhang, M. Li, Y. Zhou, B. Lu, D. Yan, M. Wei, D. G. Evans and X. Duan, Localization of Au Nanoclusters on Layered Double Hydroxides Nanosheets: Confinement-Induced Emission Enhancement and Temperature-Responsive Luminescence, Adv. Funct. Mater., 2015, 25, 5006-5015.

19 (a) W. Guan, J. Lu, W. Zhou and C. Lu, Aggregation-induced Emission Molecules in Layered Matrices for Two-color Luminescence Films, Chem. Commun., 2014, 50, 11895-11898; (b) H. Zhou, Q. Ye, W. T. Neo, J. Song, H. Yan, Y. Zong, B. Z. Tang, T. S. A. Hor and J. Xu, Electrospun Aggregationinduced Emission Active POSS-based Porous Copolymer Films for Detection of Explosives, Chem. Commun., 2014, 50, 13785-13788; (c) Z. Li, R. Liang, W. Liu, D. Yan and M. Wei, A Dual-stimuli-Responsive Fluorescent Switch Ultrathin Film, Nanoscale, 2015, 7, 16737-16743; (d) Z. Ma, C. Liu, N. Niu, Z. Chen, S. Li, S. Liu and J. Li, Seeking Brightness from Nature: J. Aggregation-Induced Emission in Cellulolytic Enzyme Lignin Nanoparticles, ACS Sustainable Chem. Eng., 2018, 6, 3169-3175. 20 M. Yuan, D. Wang, P. Xue, W. Wang, J. Wang, Q. Tu, Z. Liu, Y. Liu, Y. Zhang and J. Wang, Fluorenone Organic Crystals: 
Two-Color Luminescence Switching and Reversible Phase Transformations between $\pi-\pi$ Stacking-Directed Packing and Hydrogen Bond-Directed Packing, Chem. Mater., 2014, 26, 2467-2477.

21 (a) D. E. Barlow, G. H. Dickinson, B. Orihuela, J. L. Kulp, D. Rittschof and K. J. Wahl, Characterization of the Adhesive Plaque of the Barnacle Balanus Amphitrite: Amyloid-like Nanofibrils are a Major Component, Langmuir, 2010, 26, 6549-6556; (b) J. Gu, S. Miao, Z. Yan and P. Yang, Multiplex Binding of Amyloid-like Protein Nanofilm to Different Material Surfaces, Colloid Interface Sci. Commun., 2018, 22, 42-48.

22 R. I. Litvinov, D. A. Faizullin, Y. F. Zuev and J. W. Weisel, The $\alpha$-Helix to $\beta$-Sheet Transition in Stretched and Compressed Hydrated Fibrin Clots, Biophys. J., 2012, 103, 1020-1027.

23 G. Wei, Z. Su, N. P. Reynolds, P. Arosio, I. W. Hamley, E. Gazit and R. Mezzenga, Self-assembling Peptide and Protein Amyloids: From Structure to Tailored Function in Nanotechnology, Chem. Soc. Rev., 2017, 46, 4661-4708.

24 C. Reichhardt, A. N. Jacobson, M. C. Maher, J. Uang, O. A. McCrate, M. Eckart and L. Cegelski, Congo Red Interactions With Curli-producing E. coli and Native Curli Amyloid Fibers, PLoS One, 2015, 10, e0140388.

25 (a) H. Naiki, K. Higuchi, M. Hosokawa and T. Takeda, Fluorometric Determination of Amyloid Fibrils in Vitro Using the Fluorescent Dye, Thioflavine T, Anal. Biochem., 1989, 177, 244-249; (b) H. LeVine, Thioflavine T interaction With Amyloid $\beta$-Sheet Structures, Amyloid: Int. J. Exp. Clin. Invest., 1995, 2, 1-6; (c) S. Chimon and Y. Ishii, Capturing Intermediate Structures of Alzheimer's $\beta$-Amyloid, $A \beta(1-40)$, by Solid-State NMR Spectroscopy, J. Am. Chem. Soc., 2005, 127, 13472-13473.

26 (a) E. E. Nesterov, J. Skoch, B. T. Hyman, W. E. Klunk, B. J. Bacskai and T. M. Swager, In Vivo Optical Imaging of Amyloid Aggregates in Brain: Design of Fluorescent Markers, Angew. Chem., Int. Ed., 2005, 44, 5452-5456; (b) M. S. Celej, E. A. Jares-Erijman and T. M. Jovin, Fluorescent N-Arylaminonaphthalene Sulfonate Probes for Amyloid Aggregation of a-Synuclein, J. Biophys., 2008, 94, 4867-4879; (c) W. M. Chang, M. Dakanali, C. C. Capule, C. J. Sigurdson, J. Yang and E. A. Theodorakis, ANCA: A Family of Fluorescent Probes that Bind and Stain Amyloid Plaques in Human Tissue, ACS Chem. Neurosci., 2011, 2, 249-255.

27 (a) G. Gorbenko, V. Trusova, E. Kirilova, G. Kirilov, I. Kalnina, A. Vasilev, S. Kaloyanova and T. Deligeorgiev, New fluorescent probes for detection and characterization of amyloid fibrils, Chem. Phys. Lett., 2010, 495, 275-279; (b) Q. Huang, J. Xie, Y. Liu, A. Zhou and J. Li, Detecting the Formation and Transformation of Oligomers during Insulin Fibrillation by a Dendrimer Conjugated with Aggregation-Induced Emission Molecule, Bioconjugate Chem., 2017, 28, 944-956.

28 (a) W. Fu, C. Yan, Z. Guo, J. Zhang, H. Zhang, H. Tian and W. Zhu, Rational Design of Near-Infrared AggregationInduced-Emission-Active Probes: In Situ Mapping of Amyloid- $\beta$ Plaques with Ultrasensitivity and High-Fidelity, J. Am. Chem. Soc., 2019, 141, 3171-3177; (b) J. Zhang, J. Mei, X. Hu, X. He and H. Tian, Ratiometric Detection of $\beta$-Amyloid and Discrimination from Lectins by a Supramolecular AIE Glyconanoparticle, Small, 2016, 12, 6562-6567; (c) N. Pradhan, D. Jana, B. K. Ghorai and N. R. Jana, Detection and Monitoring of Amyloid Fibrillation Using a Fluorescence "Switch-On" Probe, ACS Appl. Mater. Interfaces, 2015, 7, 25813-25820.

29 (a) W. E. Klunk, J. W. Pettegrew and D. J. Abraham, Quantitative Evaluation of Congo Red Binding to Amyloid-like Proteins With a Beta-pleated Sheet Conformation, J. Histochem. Cytochem., 1989, 37, 1273-1281; (b) W. E. Klunk, M. L. Debnath and J. W. Pettegrew, Chrysamine-G Binding to Alzheimer and Control Brain: Autopsy Study of a New Amyloid Probe, Neurobiol. Aging, 1995, 16, 541-548; (c) I. Roterman, M. Krol, M. Nowak, L. Konieczny, J. Rybarska, B. Stopa, B. Piekarska and G. Zemanek, Why Congo Red Binding is Specific for Amyloid Proteins-model Studies and A Computer Analysis Approach, Med. Sci. Monit., 2001, 7, 771-784.

30 J. Xie, M. Qin, Y. Cao and W. Wang, Mechanistic Insight of Photo-induced Aggregation of Chicken Egg White Lysozyme: The Interplay Between Hydrophobic Interactions and Formation of Intermolecular Disulfide Bonds, Proteins, 2011, 79, 2505-2516.

31 Y. Ha, J. Yang, F. Tao, Q. Wu, Y. Song, H. Wang, X. Zhang and P. Yang, Phase-transited Lysozyme as a universal route to bioactive hydroxyapatite crystalline film, Adv. Funct. Mater., 2018, 28, 1704476.

32 H. Mills, S. Skodbo and P. Blyth, Understanding Organised Crime: Estimating The Scale and The Social and Economic Costs, Res. Rep., 2013, 73, 1-137.

33 S. Verma, R. Kumar and P. J. Philip, Economic and Societal Impact of Global Counterfeiting and Piracy, Pac. Bus. Rev. Int., 2014, 6, 98-104.

34 J. Gooch, B. Danie, V. Abbate and N. Frascione, Taggant Materials in Forensic Science: A Review, Trends Anal. Chem., 2016, 83, 49-54.

35 (a) P. Aldhous, Murder by Medicine, Nature, 2005, 434, 132-136; (b) D. Bansal, S. Malla, K. Gudala and P. Tiwari, Anti-Counterfeit Technologies: A Pharmaceutical Industry Perspective, Sci. Pharm., 2013, 81, 1-13.

36 (a) A. K. Deisingh, Pharmaceutical Counterfeiting, Analyst, 2005, 130, 271-279; (b) N. Zadbuke, S. Shahi, B. Gulecha, A. Padalkar and M. Thube, Recent Trends and Future of Pharmaceutical Packaging Technology, J. Pharm. Bioanal. Sci., 2013, 5, 98-110; (c) T. K. Mackey and G. Nayyar, A review of Existing and Emerging Digital Technologies to Combat the Global Trade in Fake Medicines, Expert Opin. Drug Saf., 2017, 16, 587-602.

37 X. Pang, L. Shi, J. Song, X. Chen and S. Chen, Use of The Potential DNA Barcode ITS2 to Identify Herbal Materials, J. Nat. Med., 2013, 67, 571-575.

38 (a) J. Andres, R. D. Hersch, J. Moser and A. Chauvin, A New Anti-Counterfeiting Feature Relying on Invisible Luminescent Full Color Images Printed with Lanthanide-Based Inks, $A d v$. Funct. Mater., 2014, 24, 5029-5036; (b) Y. Liu, L. Zhou, Y. Li, R. Deng and H. Zhang, Highly Fluorescent Nitrogen-doped Carbon Dots with Excellent Thermal and Photo Stability Applied as Invisible Ink for Loading Important Information 
and Anti-counterfeiting, Nanoscale, 2017, 9, 491-496; (c) N. Kanika, P. Kumar, S. Singh and B. K. Gupta, A Novel Approach to Synthesise a Dual-Mode Luminescent Composite Pigment for Uncloneable High-Security Codes to Combat Counterfeiting, Chem. - Eur. J., 2017, 23, 17144-17151.

39 (a) Z. An, C. Zheng, Y. Tao, R. Chen, H. Shi, T. Chen, Z. Wang, H. Li, R. Deng, X. Liu and W. Huang, Stabilizing Triplet Excited States for Ultralong Organic Phosphorescence, Nat. Mater., 2015, 14, 685-690; (b) M. Tsang, G. Bai and J. Hao, Stimuli Responsive Upconversion Luminescence Nanomaterials and Films for Various Applications, Chem. Soc. Rev., 2015, 44, 1585-1607; (c) R. Gao, D. Yan, D. G. Evans and X. Duan, Layer-by-layer Assembly of Longafterglow Self-supporting Thin Films with Dual-stimuli-responsive Phosphorescence and Antiforgery Applications, Nano Res., 2017, 10, 3606-3617; (d) H. Tan, S. Xie, N. Li, C. Tong, L. Xu, J. Xu and C. Zhang, Synthesis and Characterization of NaYF4: Yb,Er Up-conversion Phosphors/poly(vinyl alcohol) Composite Fluorescent Films, Mater. Express, 2018, 8, 141-148.

40 (a) Y. Deng, D. Zhao, X. Chen, F. Wang, H. Song and D. Shen, Long Lifetime Pure Organic Phosphorescence Based on Water Soluble Carbon dots, Chem. Commun., 2013, 49, 5751-5753; (b) K. Jiang, L. Zhang, J. Lu, C. Xu, C. Cai and H. Lin, TripleMode Emission of Carbon Dots: Applications for Advanced AntiCounterfeiting, Angew. Chem., Int. Ed., 2016, 55, 7231-7235; (c) S. Lin, Y. Cheng, C. Lin, J. Fang, W. Xiang and X. Liang, Carbon Nanodots with Intense Emission From Green to Red and Their Multifunctional Applications, J. Alloys Compd., 2018, 742, 212-219.

41 C. Zhang, B. Wang, W. Li, S. Huang, L. Kong, Z. Li and L. Li, Conversion of Invisible Metal-organic Frameworks to Luminescent Perovskite Nanocrystals for Confidential Information Encryption and Decryption, Nat. Commun., 2017, 8, 1138-1147.

42 (a) F. Fayazpour, B. Lucas, N. Huyghebaert, K. Braeckmans, S. Derveaux, B. G. Stubbe, J. Remon, J. Demeester, C. Vervaet and S. C. De, Smedt, Encoded Drug Tablets to Combat Counterfeiting, Adv. Mater., 2007, 19, 3854-3858; (b) C. Huang, B. Lucas, C. Vervaet, K. Braeckmans, S. Van. Calenbergh, I. Karalic, M. Vandewoestyne, D. Deforce, J. Demeester and S. C. De, Smedt, Unbreakable Codes in Electrospun Fibers: Digitally Encoded Polymers to Stop Medicine Counterfeiting, Adv. Mater., 2010, 22, 26572662; (c) C. Huang, S. J. Soenen, J. Rejman, B. Lucas, K. Braeckmans, J. Demeestera and S. C. De, Smedt, Stimuli-responsive Electrospun Fibers and Their Applications, Chem. Soc. Rev., 2011, 40, 2417-2434.

43 (a) J. Hou, M. Li and Y. Song, Patterned Colloidal Photonic Crystals, Angew. Chem., Int. Ed., 2018, 57, 2544-2553; (b) Z. Gao, C. Huang, D. Yang, H. Zhang, J. Guo and J. Wei, Dual-mode Multicolored Photonic Crystal Patterns Enabled by Ultravioletresponsive Core-shell Colloidal Spheres, Dyes Pigm., 2018, 148, 108-117; (c) K. Liu, Y. Tian, Q. Li, X. Du, J. Zhang, C. Wang and S. Chen, Microfluidic Printing Directing Photonic Crystal Bead 2D Code Patterns, J. Mater. Chem. C, 2018, 6, 2336-2341.

44 (a) J. Kim, J. M. Yun, J. Jung, H. Song, J. Kim and H. Ihee, Anti-Counterfeit Nanoscale Fingerprints Based on Randomly
Distributed Nanowires, Nanotechnology, 2014, 25, 155303155310; (b) A. F. Smith, P. Patton and S. E. Skrabalak, Plasmonic Nanoparticles as a Physically Unclonable Function for Responsive Anti-Counterfeit Nanofingerprints, Adv. Funct. Mater., 2016, 26, 1315-1321.

45 (a) A. L. Schmucker, M. B. Dickerson, M. Rycenga, B. F. Mangelson, K. A. Brown, R. R. Naik and C. A. Mirkin, Combined Chemical and Physical Encoding with Silk Fibroin-Embedded Nanostructures, Small, 2014, 10, 1485-1489; (b) O. Poncelet, G. Tallier, P. Simonis, A. Cornet and L. A. Francis, Synthesis of Bio-inspired Multilayer Polarizers and Their Application to Anti-counterfeiting, Bioinspiration Biomimetics, 2015, 10, 026004-026014; (c) Y. Wu, Y. Zhong, B. Chu, B. Sun, B. Song, S. Wu, Y. Su and Y. He, Plant-derived Fluorescent Silicon Nanoparticles Featuring Excitation Wavelength-dependent Fluorescence Spectra for Anti-counterfeiting Applications, Chem. Commun., 2016, 52, 7047-7050.

46 (a) B. Duong, H. Liu, L. Ma and M. Su, Covert Thermal Barcodes Based on Phase Change Nanoparticles, Sci. Rep., 2014, 4, 5170-5175; (b) Y. Cui, I. Y. Phang, Y. H. Lee, M. R. Lee, Q. Zhang and X. Ling, Multiplex Plasmonic Anti-counterfeiting Security Labels Based on Surface-enhanced Raman Scattering, Chem. Commun., 2015, 51, 5363-5366; (c) M. Qin, M. Sun, R. Bai, Y. Mao, X. Qian, D. Sikka, Y. Zhao, H. Qi, Z. Suo and X. He, Bioinspired Hydrogel Interferometer for Adaptive Coloration and Chemical Sensing, Adv. Mater., 2018, 30, 1800468.

47 (a) K. P. Pfeuffer, J. Caldwell, J. T. Shelley, S. J. Ray and G. M. Hieftje, Detection of Counterfeit Electronic Components Through Ambient Mass Spectrometry and Chemometrics, Analyst, 2014, 139, 4505-4511; (b) K. Dégardin, Y. Roggo and P. Margot, Forensic Intelligence for Medicine Anti-counterfeiting, Forensic Sci. Int., 2015, 248, 15-32.

48 (a) B. Berman, Strategies to Detect and Reduce Counterfeiting Activity, Bus. Hori., 2008, 51, 191-199; (b) E. L. Prime and D. H. Solomon, Australia's Plastic Banknotes: Fighting Counterfeit Currency, Angew. Chem., Int. Ed., 2010, 49, 3726-3736; (c) R. Li, Y. Zhang, J. Tan, J. Wan, J. Guo and C. Wang, Dual-Mode Encoded Magnetic Composite Microsphere Based on Fluorescence Reporters and Raman Probes as Covert Tag for Anticounterfeiting Applications, ACS Appl. Mater. Interfaces, 2016, 8, 9384-9394.

49 X. Goh, Y. Zheng, S. J. Tan, L. Zhang, K. Kumar, C. Qiu and J. K. W. Yang, Three-dimensional Plasmonic Stereoscopic Prints in Full Colour, Nat. Commun., 2014, 5, 5361-5369.

50 J. Lee, P. W. Bisso, R. L. Srinivas, J. J. Kim, A. J. Swiston and P. S. Doyle, Universal Process-inert Encoding Architecture for Polymer Microparticles, Nat. Mater., 2014, 13, 524-530.

51 M. R. Carro-Temboury, R. Arppe, T. Vosch and T. J. Sørensen, An Optical Authentication System Based on Imaging of Excitationselected Lanthanide Luminescence, Sci. Adv., 2018, 4, 1-8.

52 P. Kumar, S. Singh and B. K. Gupta, Future Prospects of Luminescent Nanomaterial Based Security Inks: From Synthesis to Anti-counterfeiting Applications, Nanoscale, 2016, 8, 14297-14340. 